\title{
Adjunctive Middle Turbinectomy for Endoscopic Medial Orbital Wall - Thyroid Eye Disease Decompression
}

\author{
Andrea A. Tooley ${ }^{1}$ (1) \\ Matthew Kim ${ }^{3}$ \\ Ann Q. Tran ${ }^{2}$ \\ ${ }^{1}$ Department of Ophthalmology, Mayo Clinic, Rochester, Minnesota, \\ USA \\ 2 Oculoplastic and Orbital Surgery, Edward S. Harkness Eye Institute, \\ Columbia University Irving Medical Center, New York, New York \\ ${ }^{3}$ Department of Otolaryngology - Head and Neck Surgery, Division of \\ Rhinology and Anterior Skull Base Surgery, New York-Presbyterian \\ Hospital, New York, New York
}

Int Arch Otorhinolaryngol 2022;26(4):e579-e584.

\author{
Michael Kazim² David A. Gudis ${ }^{3}$
}

Address for correspondence Andrea A. Tooley, MD, Mayo Clinic, 200 First St. SW, Rochester, MN 55905 (e-mail: Tooley.andrea@mayo.edu).

\begin{abstract}
Keywords

- Middle turbinate

- orbital decompression

- thyroid eye disease

Introduction Middle turbinate resection (MTR) is commonly performed during endonasal endoscopic sinus and skull base surgery.

Objective The purpose of this study was to characterize the additional orbital softtissue volume expansion during endoscopic medial orbital wall decompression with adjunctive MTR.

Methods A retrospective review of patients who underwent endoscopic medial wall decompression with MTR was performed. The imaging software AW (GE Healthcare, Chicago, IL, USA) was used to overlay pre and postoperative orbital computed tomography $(\mathrm{CT})$ images to visualize the preoperative position of the middle turbinate and the postoperative orbital soft tissue in the ethmoid bed. The imaging software Vitrea (Vital Images Inc., Minnetonka, MN, USA) was used to manually segment postoperative scans to determine the volume of orbital tissue which had filled the space previously occupied by the middle turbinate or medial to it.

Results Nine orbits from 5 patients were included in this study; all patients were female with a history of hyperthyroidism. The average age was 55.6 years (range 3274). Of the 9 orbits, 7 (78\%) had orbital soft tissue within the space of the resected middle turbinate postoperatively. The average volume of orbital tissue within or medial to this space was $0.83+/-0.67 \mathrm{cc}$. No patients had any postoperative complications. Conclusions In this patient cohort, adjunctive middle turbinate resection for endoscopic medial orbital wall decompression added $\sim 0.83$ cc of volume for orbital soft tissue after medial wall decompression. Middle turbinate resection is a useful adjunct to the orbital surgeon's armamentarium to augment the results of a medial orbital decompression for select patients.
\end{abstract}

received

December 18, 2020 accepted after revision

May 7, 2021

published online

February 8, 2022
DOI https://doi.org/ 10.1055/s-0041-1740156. ISSN 1809-9777. (c) 2022. Fundação Otorrinolaringologia. All rights reserved.

This is an open access article published by Thieme under the terms of the Creative Commons Attribution-NonDerivative-NonCommercial-License, permitting copying and reproduction so long as the original work is given appropriate credit. Contents may not be used for commercial purposes, or adapted, remixed, transformed or built upon. (https://creativecommons.org/ licenses/by-nc-nd/4.0/)

Thieme Revinter Publicações Ltda., Rua do Matoso 170, Rio de Janeiro, RJ, CEP 20270-135, Brazil 


\section{Introduction}

Orbital decompression for thyroid eye disease (TED) is performed to increase the boney orbital volume to accommodate the pathologically expanded orbital soft tissues. Traditional indications for orbital decompression include compressive optic neuropathy (CON) or extreme proptosis producing disfigurement or corneal exposure. ${ }^{1}$ Orbital decompression may be customized to account for the unique patient's anatomy, orbital pathology, and surgical goals. ${ }^{2}$ The endonasal endoscopic approach to medial orbital decompression provides excellent visualization of the medial aspect of the orbit, including the apex and floor medial to the infraorbital nerve, without the need for an external incision. In the standard surgical approach, the ethmoidal air cells and lamina papyracea are removed in addition to the medial aspect of the floor.

To maximize the decompressive effect of a two-wall approach (medial wall and floor), we have previously advised removal of the posterior $2 / 3$ of the orbital strut. ${ }^{3}$ We now describe the addition of a middle turbinectomy to further augment the medial expansion of orbital contents, and, in turn, the decompressive outcome. Middle turbinate resection (MTR) is commonly performed during endonasal endoscopic sinus and skull base surgery for inflammatory and neoplastic disorders. While the increase in orbital contents expansion after standard endoscopic decompression has been well established, ${ }^{3-6}$ adjunctive MTR to maximize medial wall decompression has not previously been described. In addition to improved visualization, MTR may allow additional expansion of orbital contents into the ethmoid bed.

Herein, we present a volumetric analysis of the orbital soft-tissue expansion into the ethmoid bed after endoscopic medial wall decompression with MTR to quantify the volume of expansion into space previously occupied or obstructed by the middle turbinate.

\section{Methods}

Institutional Review Board approval was obtained for this Health Insurance Portability and Accountability Act compliant study, which adheres to the tenants of the Declaration of Helsinki. A retrospective review of patients who underwent endoscopic medial wall decompression with MTR between July 2019 and April 2020 was performed. All endonasal surgeries were performed by a single surgeon (D. G.) and additional orbital surgeries, including lateral wall decompressions and upper eyelid retraction repair, were performed by M. K. The inclusion criteria were patients with thyroid eye disease in the active or quiescent phase, and patients with pre and postoperative orbital computed tomography (CT) imaging available. The exclusion criteria were patients who had previous endonasal surgery.

\section{Surgical Technique}

The surgery begins with ipsilateral comprehensive endoscopic sinus surgery, including a maxillary antrostomy, sphenoid sinusotomy, total ethmoidectomy, and frontal sinusotomy. Next, a curved endoscopic scissor is used to resect the entire middle turbinate from the axilla to the basal lamella up to its skull base attachment, with the curve of the scissor aimed inferiorly to avoid skull base injury as the dissection proceeds posteriorly. Care should be taken to avoid injury to the olfactory region and skull base. Suction monopolar cautery is used for hemostasis at the basal lamella arterial branch of the sphenopalatine artery. Next, the mucosa overlying the medial orbital wall is removed, and curettes are used to fracture and remove the lamina papyracea and orbital floor medial to the infraorbital canal. In some patients, a 4-mm diamond burr drill is then used to resect the orbital process of the palatine bone as well. Next, a disposable Beaver blade and arthroplasty meniscus blade are used to incise periorbita. A ball-tipped maxillary ostium seeker is used to separate the periorbita from the orbital fat and muscle, and grasping forceps are then used to remove the periorbita.

\section{Image Analysis}

Imaging software AW (GE Healthcare, Chicago, IL, USA) was used to overlay pre- and postoperative orbital CT scans in the axial and coronal planes. The window level $(0-100 \%)$ of each scan was adjusted to visualize the preoperative position of the middle turbinate and the postoperative orbital soft tissue that had prolapsed into the ethmoid sinus ( - Fig. $\mathbf{1}$ ). The
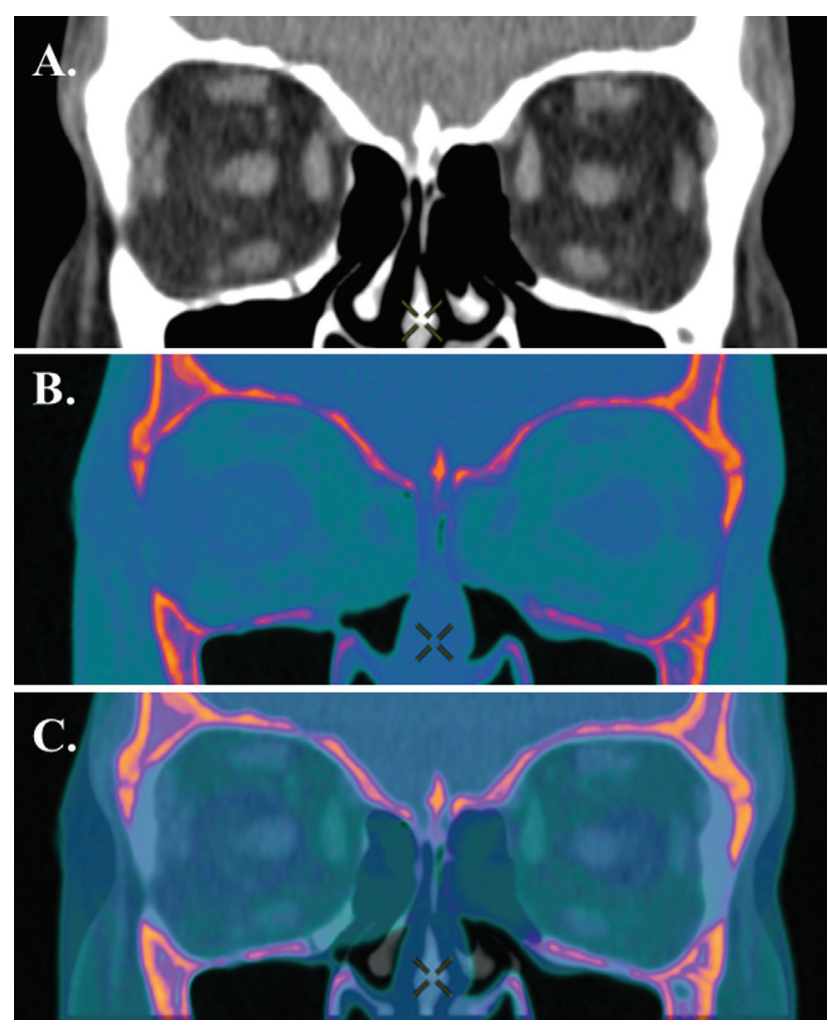

Fig. 1 Patient 3. Fusion of pre and postoperative orbital computer tomography scans. (A) Preoperative coronal computer tomography. (B) Postoperative coronal computer tomography after bilateral medial wall decompression and middle turbinate resection. Note herniated orbital soft tissue up to the nasal septum. (C) Fusion of pre and postoperative scan, each at $50 \%$ opacity. 


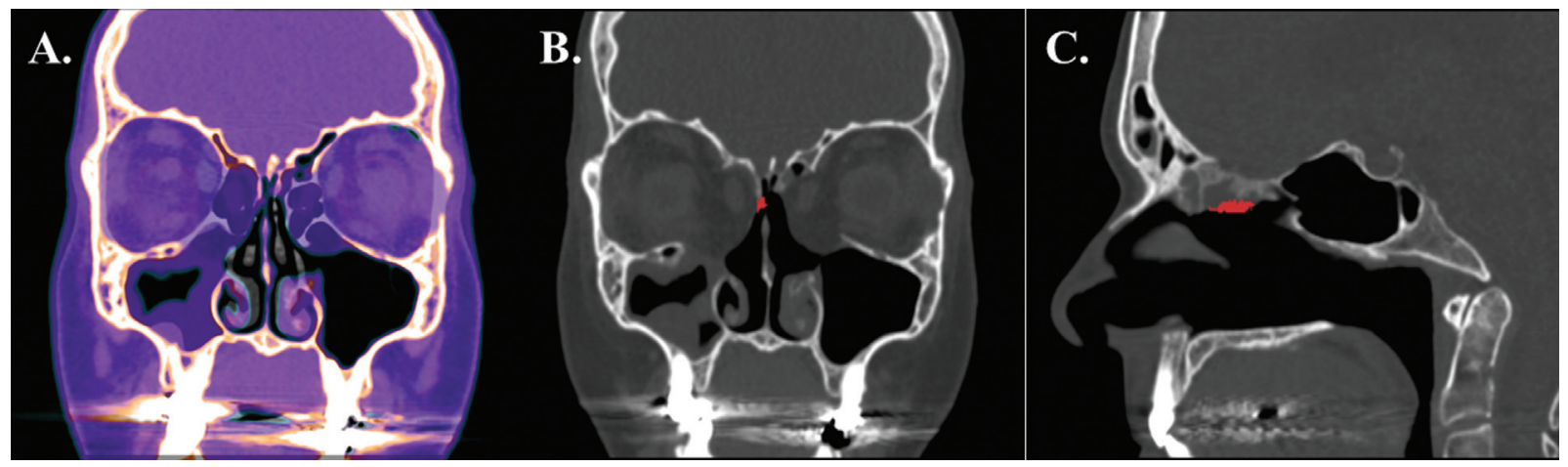

Fig. 2 Patient 4. (A) Fusion of pre and postoperative coronal computer tomography scan. (B) Red shading represents volumetric segmentation of small amount of orbital soft tissue prolapsed into the space previously occupied by the right middle turbinate. Note that no orbital soft tissue has prolapsed into this space on the left. (C) Sagittal view of orbital soft tissue within the space of the resected middle turbinate, shaded in red.

imaging software Vitrea (Vital Images Inc., Minnetonka, MN, USA) was used to manually segment postoperative scans to determine the volume of orbital tissue which had filled the space previously occupied by or medial to the middle turbinate. Only orbital tissue within the space of the middle turbinate or medial up to the nasal septum was included in the volume analysis (-Fig. 2). Volumetric analysis was performed on each segmented side individually ( - Fig. 3 ).

Comparisons of middle turbinate volume were made using the Mann-Whitney U test.

\section{Results}

Nine orbits from 5 patients were included in this study. All patients were female, with an average age of 55.6 years (range 32-74). Patient demographics are displayed in -Table 1. All patients had a history of hyperthyroidism and were euthyroid at the time of surgery. Indications for decompression included CON (40\%), as evidenced by visual acuity, pupillary reactions, color vision, and Humphrey visual field testing, and excessive proptosis with corneal exposure/ulceration (60\%). Patients had a combination of orbital fat and extraocular muscle enlargement. All patients underwent endonasal medial wall and floor decompression. Two patients (4 orbits) underwent concurrent lateral wall and fat decompression. Two of five patients (both with $\mathrm{CON}$ ) were treated with orbital radiotherapy postoperatively and $4 / 5$ patients were treated with steroids, either intravenous or oral, perioperatively.

Pre and postoperative exam details are listed in - Table 1. Preoperative BCVA was $20 / 30$ or better in $3 / 5$ patients. Two patients had decreased acuity secondary to CON or keratopathy. Postoperatively, vision remained stable or improved in all patients. All patients received postoperative imaging
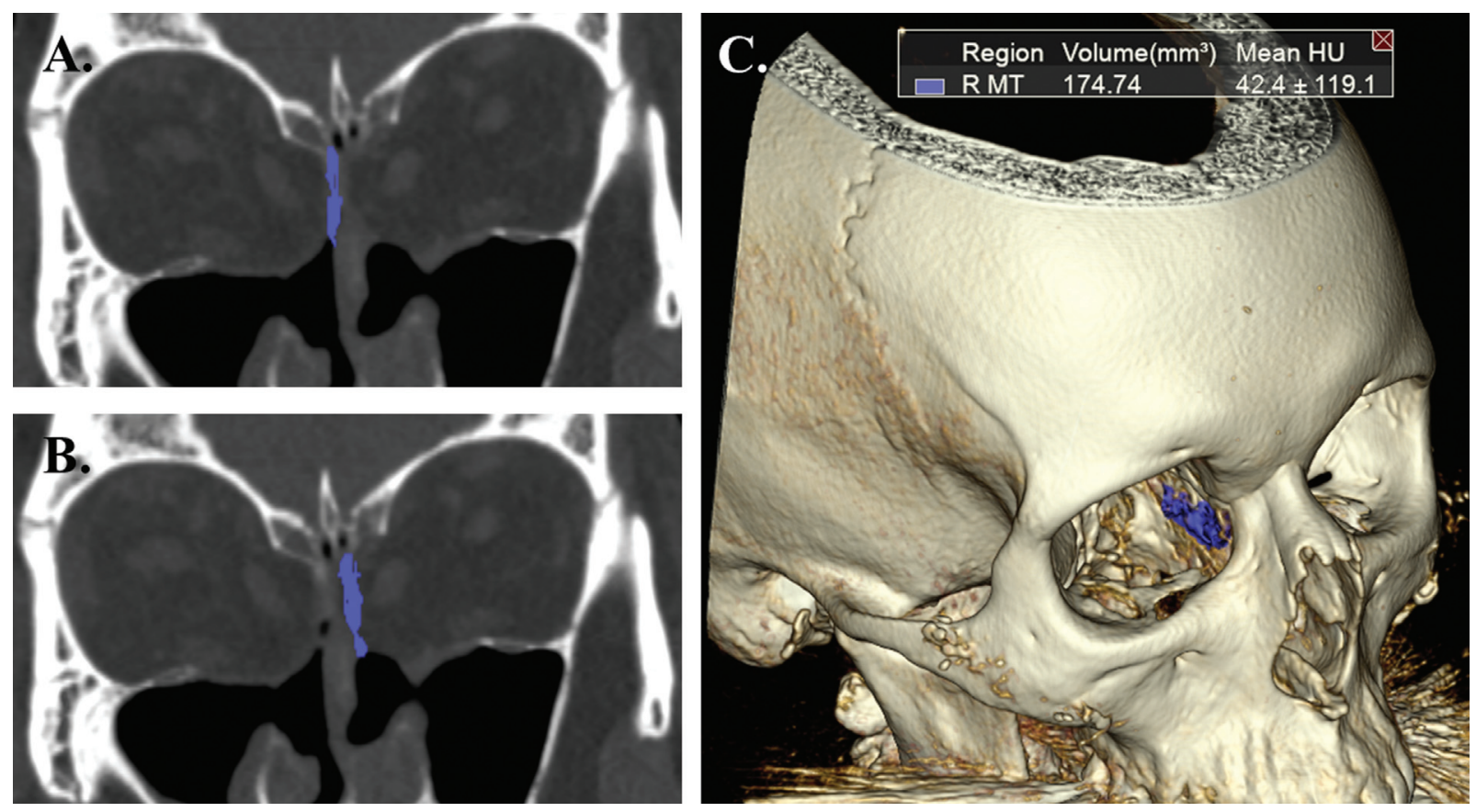

Fig. 3 Patient 1. (A) and (B) Postoperative coronal computer tomography showing volumetric segmentation of orbital soft tissue within the area of the resected middle turbinate on the right and left, shaded in blue. (C) 3D reconstruction of orbital soft tissue within the space of the resected middle turbinate and volumetric analysis showing $\sim 1.74 \mathrm{cc}$ of volume in this region on the right, shaded in blue. 


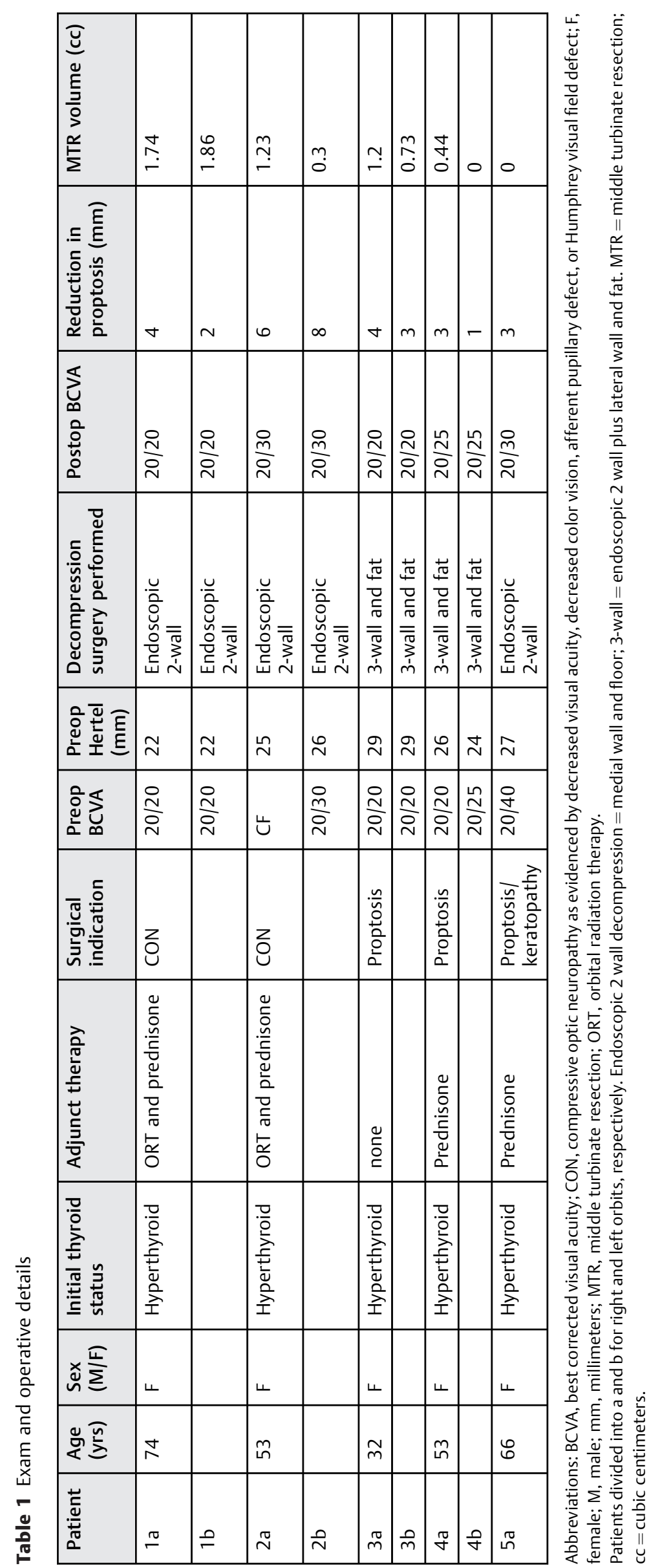


within 1 month following surgery. The follow-up time ranged from 4 to 9 months.

Of the 9 orbits, 7 (78\%) had orbital soft tissue occupying the space of the resected middle turbinate evident on postoperative imaging. The average volume of orbital tissue within or medial to the space previously occupied by the middle turbinate was $0.83+/-0.67 \mathrm{cc}$. The range of volume for the $7 / 9$ patients with orbital soft-tissue expansion into the middle turbinate space was 0.3 to $1.9 \mathrm{cc}$. Two orbits did not have soft tissue prolapse into the space of the resected middle turbinate. Volume did not differ between patients who had undergone 2 or 3 -wall decompressions, $p=0.41$.

The average pre-operative Hertel exophthalmometry was $25.5 \mathrm{~mm}$ and $25.6 \mathrm{~mm}$ on the right and left respectively (range 22-29). The average postoperative Hertel exophthalmometry was $22 \mathrm{~mm}$ and $22.5 \mathrm{~mm}$ (range 18-26). The average improvement in Hertel exophthalmometry was $3.78 \mathrm{~mm}$ (range $1-8 \mathrm{~mm}) p=0.006$.

No patients suffered from any complications related to middle turbinectomy, which may include postoperative epistaxis, cerebrospinal fluid (CSF) rhinorrhea, atrophic rhinitis, or olfactory dysfunction.

\section{Discussion}

Endoscopic medial wall decompression for patients with thyroid eye disease (TED) is a well described procedure with documented reduction in proptosis ranging between 2 and $6 \mathrm{~mm}$ for the medial wall and floor. ${ }^{3-8}$ In a traditional endoscopic medial wall decompression, the medial anatomic limit of the decompression is the middle turbinate; MTR moves this limit medially to the nasal septum. We found that MTR in conjunction with medial wall and floor decompression increased the volume for orbital soft tissue prolapse by 0.3 to $1.9 \mathrm{cc}$ in $78 \%$ of patients. On average, each cubic centimeter of volume expansion is associated with a proptosis reduction of $1 \mathrm{~mm} .^{9}$ Therefore, we would anticipate an average of $0.83 \mathrm{~mm}$ improvement above the standard surgical results with the addition of MTR. The authors specifically segmented only orbital soft tissue within the space of the middle turbinate, and not the volume of the middle turbinate itself, to analyze the additional soft tissue space afforded by MTR. Differences in orbital decompression after MTR may be related to orbital compliance, or other patient-specific factors.

Partial middle turbinate resection is commonly performed to improve the intraoperative view during functional endoscopic sinus surgery (FESS) and also as a treatment for nasal airway obstruction (NAO) in patients with concha bullosa. ${ }^{10}$ Middle turbinate resection remains a subject of controversy among sinus surgeons, with proponents touting improvements in postoperative sinus patency and irrigation access, while opponents emphasize the risk of complications. ${ }^{11,12}$ While the middle turbinate does play physiological roles in air conditioning, sinus ventilation, air filtration, and olfaction, recent literature suggests that the rate of complications-including epistaxis, CSF leak, frontal sinus stenosis, olfactory dysfunction, and empty nose syndrome- are low. ${ }^{13-16}$ Complications of turbinate resection including atrophic rhinitis and empty nose syndrome are more often associated with total inferior turbinectomy than with partial MTR. ${ }^{13,17}$ In a series of endoscopic tumor resections and skull base reconstructions, turbinate resection was not correlated with nasal obstruction, poor sinonasal function or poor sleep. Additionally, no cases of debilitating sinonasal dysfunction or empty nose syndrome were reported. ${ }^{18}$

Middle turbinate resection has been described in endoscopic orbital decompression but is not widely or routinely utilized. ${ }^{19}$ Because MTR is an adjunct to a traditional endoscopic medial wall decompression, we do not expect outcomes or complication rates to differ substantially from those previously published and within surgical standards. ${ }^{3}$ Differences in postoperative proptosis reduction and MTR volume may be explained by variability in orbital soft-tissue fibrosis, which may limit the expansion of orbital soft tissues into the surrounding open sinonasal space. Patients 2 and 4 were shown to have side-to-side asymmetry of the volume of orbital tissue within the space of the resected middle turbinates. This may similarly be related to differences in orbital soft tissue compliance or variations in the amount of partial resection on the middle turbinate on each side of the patient.

The present study has several limitations. The small sample size and lack of a control group limit the analysis. However, $78 \%$ of the orbits in this study received augmented volume after MTR. Therefore, these findings may apply to the larger population of patients undergoing endoscopic medial wall decompression. Two imaging platforms were used in this study, AW to fuse the pre and postoperative images, and Vitrea to conduct volumetric analysis, which introduces human error. The purpose of the study, however, was to determine whether MTR augments medial wall decompression, and not to perform a detailed volumetric analysis. Further investigations with larger groups of patients as well as control groups and prolonged follow-up times may allow us to characterize the exact volume of the middle turbinate and the potential for orbital volume augmentation and proptosis reduction after resection. Two patients (4 orbits) underwent concurrent lateral wall and fat decompression; however, this did not significantly affect the overall augmented volume achieved by MTR compared with those patients undergoing only a 2-wall decompression. Additionally, 2 patients (patients 1 and 2) were within the active phase of thyroid eye disease at the time of decompression. This also did not affect outcomes or volumes achieved from MTR.

\section{Conclusion}

This preliminary study suggests that MTR augments the orbital volume expansion achieved by medial orbital decompression. Middle turbinate resection may be considered as a low-risk, useful adjunct to medial wall decompression in select patients who require maximal decompressive effect.

\section{Financial Disclosures}

None. 
584 Adjunctive Middle Turbinectomy for Endoscopic Medial Orbital Wall Tooley et al.

\section{Meeting Presentations}

None.

\section{Conflict of Interests}

The authors have no conflict of interests to declare.

\section{References}

1 Bartalena L, Pinchera A, Marcocci C. Management of Graves' ophthalmopathy: reality and perspectives. Endocr Rev 2000;21 (02):168-199

2 Tooley AA, Godfrey KJ, Kazim M. Evolution of thyroid eye disease decompression-dysthyroid optic neuropathy. Eye (Lond) 2019;33 (02):206-211

3 Finn AP, Bleier B, Cestari DM, et al. A Retrospective Review of Orbital Decompression for Thyroid Orbitopathy with Endoscopic Preservation of the Inferomedial Orbital Bone Strut. Ophthal Plast Reconstr Surg 2017;33(05):334-339

4 Kennedy DW, Goodstein ML, Miller NR, Zinreich SJ. Endoscopic transnasal orbital decompression. Arch Otolaryngol Head Neck Surg 1990;116(03):275-282

5 Stiglmayer N, Mladina R, Tomić M, et al. Endonasal endoscopic orbital decompression in patients with Graves' ophthalmopathy. Croat Med J 2004;45(03):318-322

6 Kasperbauer JL, Hinkley L. Endoscopic orbital decompression for Graves' ophthalmopathy. Am J Rhinol 2005;19(06):603-606

7 Kacker A, Kazim M, Murphy M, Trokel S, Close LG. "Balanced" orbital decompression for severe Graves' orbitopathy: technique with treatment algorithm. Otolaryngol Head Neck Surg 2003;128 (02):228-235

8 Dubin MR, Tabaee A, Scruggs JT, Kazim M, Close LG. Image-guided endoscopic orbital decompression for Graves' orbitopathy. Ann Otol Rhinol Laryngol 2008;117(03):177-185

9 Liao SL, Huang SW. Correlation of retrobulbar volume change with resected orbital fat volume and proptosis reduction after fatty decompression for Graves ophthalmopathy. Am J Ophthalmol 2011;151(03):465-9.e1

10 Dayal A, Rhee JS, Garcia GJM. Impact of Middle versus Inferior Total Turbinectomy on Nasal Aerodynamics. Otolaryngol Head Neck Surg 2016;155(03):518-525

11 Pinther S, Deeb R, Peterson EL, Standring RT, Craig JR. Complications Are Rare From Middle Turbinate Resection: A Prospective Case Series. Am J Rhinol Allergy 2019;33(06):657-664

12 Choby GW, Hobson CE, Lee S, Wang EW. Clinical effects of middle turbinate resection after endoscopic sinus surgery: a systematic review. Am J Rhinol Allergy 2014;28(06):502-507

13 LaMear WR, Davis WE, Templer JW, McKinsey JP, Del Porto H. Partial endoscopic middle turbinectomy augmenting functional endoscopic sinus surgery. Otolaryngol Head Neck Surg 1992;107 (03):382-389

14 Mariano FC, Hamerschmidt R, Soares CMC, Moreira AT. The Middle Turbinate Resection and Its Repercussion in Olfaction with the University of Pennsylvania Smell Identification Test (UPSIT). Int Arch Otorhinolaryngol 2018;22(03):280-283

15 Tan NC, Goggin R, Psaltis AJ, Wormald PJ. Partial resection of the middle turbinate during endoscopic sinus surgery for chronic rhinosinusitis does not lead to an increased risk of empty nose syndrome: a cohort study of a tertiary practice [published online ahead of print, 2018 Apr 6]. Int Forum Allergy Rhinol 2018; 10.1002/alr.22127 Doi: 10.1002 /alr.22127

16 Miller AJ, Bobian M, Peterson E, Deeb R. Bleeding risk associated with resection of the middle turbinate during functional endoscopic sinus surgery. Am J Rhinol Allergy 2016;30(02):140-142

17 Chhabra N, Houser SM. The diagnosis and management of empty nose syndrome. Otolaryngol Clin North Am 2009;42(02):311-330, ix

18 Snyderman CH, Carrau RL, Kassam AB, et al. Endoscopic skull base surgery: principles of endonasal oncological surgery. J Surg Oncol 2008;97(08):658-664

19 Jacquesson T, Abouaf L, Berhouma M, Jouanneau E. How I do it: the endoscopic endonasal optic nerve and orbital apex decompression. Acta Neurochir (Wien) 2014;156(10):1891-1896 